\title{
ALGUNAS CARACTERISTICAS DE LAS AREAS COMERCIALES ESPAÑOLAS
}

$311: 338.984 .3(46)$

por

\section{Ignacio Ballester Ros}

SUMARIO: I. INTRODUCCION.-II. LA CUOTA DE MERCADO DE LAS AREAS Y DE LAS ZONAS COMERCIALES DE ESPAÑA: 1. CONSIDERACIONES GENERALES. 2. LA CUOTA DE MERCADO EN 1979. 3. LA VARIACION DE la cuota de mercado en las AREas comerciales en el período 1969-1979. 4. LA CUOTA DE MERCADO PER CÁPITA dE LAS AREAS COMERCIALES EN 1979.-III. LA CUOTA DE RIQUEZA ACTIVA Y EL NIVEL DE DESARROLLO DE LAS AREAS COMERCIALES ESPAÑOLAS.

\section{INTRODUCCION}

Una vez más disponemos del Anuario del Mercado Español, ahora en su decimocuarta edición, correspondiente al año 1979, que patrocina, como siempre, Banesto, y que presenta las informaciones habituales sobre geografía del mercado, con un especial análisis de las cuotas de mercado de las Areas comerciales españolas y un estudio monográfico sobre el comercio exterior de los principales productos con saldo deficitario en el mercado español; las fuentes de riqueza e instrumento del mercado con un estudio de los consumos energéticos; los índices de capacidad de compra provincial y el equipamiento comercial mayorista y minorista; los índices de riqueza activa y niveles de desarrollo de las Areas comerciales; los indicadores socioeconómicos del mercado español; el análisis de la coyuntura con determinación de los índices vivacidad y el estudio de los movimientos migratorios interiores, y finalmente la infor- 
mación estadística referida a los Municipios con población superior a los 3.000 habitantes.

Entre tan abundante información hemos escogido como materia para este comentario las Areas comerciales, por su extraordinario interés local, con el análisis de la estructura y evolución de la cuota de mercado en los últimos diez años y la cuota de riqueza activa y su nivel de desarrollo en 1977.

\section{LA CUOTA DE MERCADO DE LAS AREAS Y DE LAS ZONAS COMERCIALES DE ESPAÑA}

\section{CONSIDERACIONES GENERALES}

Como se expone en el propio Anuario del Mercado Español, la cuota de mercado es un valor objetivo que indica la cantidad de productos o servicios que, teóricamente y en igualdad de condiciones, pueden absorber las distintas zonas territoriales, en este caso las Areas comerciales, que fueron configuradas ya en el Atlas Comercial de España, publicado por el Consejo Superior de Cámaras de Comercio, Industria y Navegación de España, y elaborado por el mismo equipo de estadísticos y economistas que vienen elaborando, bajo el patrocinio de Banesto, el citado Anuario.

Con la cuota de mercado se pretende comparar la capacidad de consumo de las Areas comerciales, de acuerdo con su volumen de población y la incidencia, más o menos intensa, del conjunto de variables que determinan la efectiva capacidad de compra de los grupos sociales insertos en cada una de ellas.

Se trata, en realidad, de un índice teórico, que constituye una medida racional y adecuada para ponderar y valorar la capacidad de consumo que cabe atribuir, en principio, a cada Area, en especial cuando se hace referencia a productos que son objeto de una distribución homogénea en el ámbito nacional; pero que presentan desviaciones en función de las características específicas de ciertos mercados y de los productos que se ofrecen en ellos en venta, ya que se considera como axiomático que existen tantos mercados como productos.

Las cuotas de mercado de cada Area comercial, que vienen elaborándose y publicándose desde el año 1966 en el repetido Anuario, se calculan partiendo de los datos contenidos en cinco series estadísticas relativas a: Población de hecho; Número de licencias co- 
merciales; Número de entidades bancarias; Número de teléfonos, incluidas las peticiones pendientes de instalación, y el Importe de la recaudación por el impuesto de espectáculos correspondiente a cada uno de los Municipios españoles.

Los citados datos municipales se agrupan por Areas comerciales y se halla el promedio de las proporciones que, en cada serie estadística, comprende a tales Areas sobre una base nacional de 100.000 unidades. La cuota de mercado general, para cada Area, se obtiene igualmente como proporción sobre 100.000 unidades, por lo que los valores respectivos deben entenderse como proporciones de consumo correspondientes a cada Area en el ámbito nacional.

A su vez, las 101 Areas comerciales se agrupan en 52 Zonas comerciales, de las que 25 están integradas por una sola Area, y las otras 27 por agrupaciones de Areas comerciales. En general, las Zonas comerciales no coinciden exactamente con las Provincias. Dos Zonas, las de Talavera de la Reina y Tortosa, se consideran independientes, dada la autonomía comercial de que han disfrutado tradicionalmente.

\section{LA CUOTA DE MERCADO EN 1979}

Dada la falta de homogeneidad en la distribución de la población española sobre el territorio y el distinto grado de desarrollo agrario, industrial y de servicios, es lógico suponer que la cuota de mercado de las Areas y Zonas comerciales ofrezca valores muy dispares entre sí, y que los más altos valores se concentren en un número reducido de aquéllas, coincidentes, en principio, con las mayores concentraciones de población y de más intensa actividad económica.

En efecto, por lo que a las Areas comerciales se refiere:

a) Madrid y Barcelona tienen las cuotas de mercado más elevadas, que en conjunto suponen el 31,1 por 100 , casi la tercera parte de la cuota total de mercado de España.

b) Valencia ocupa el tercer lugar, con el 5,1 por 100, y juntamente con Bilbao y San Sebastián, Zaragoza, Sevilla y Málaga suman un total de más de 19.000 unidades, prácticamente la quinta parte de la cuota de mercado nacional.

c) Les siguen en importancia, con una cuota superior a mil unidades, las Areas de La Coruna, Vigo, Oviedo, Santander y Pam- 
plona, al Norte; Valladolid, en la Submeseta Norte; Lérida y Gerona, Castellón, Alicante y Murcia, más Palma de Mallorca, en Cataluña y el litoral oriental mediterráneo; Córdoba y Granada, en Andalucía, y las de las dos capitales canarias. Estas Areas superan, en conjunto, las 21.000 unidades, algo más de la quinta parte de la cuota nacional de mercado.

En conjunto, las 24 Areas enumeradas absorben el 72,7 por 100 , casi las tres cuartas partes de la cuota de mercado total. Salvo Madrid y Valladolid, situadas en la Meseta, las Areas comerciales más importantes se sitúan en la periferia de aquélla, con un mayor predominio en el Norte y en el litoral mediterráneo.

Muy similar es la distribución de las Zonas comerciales más importantes, en función de su cuota de mercado. Madrid y Barcelona son las de cuota de mercado más elevada, y absorben, en conjunto, el 32,6 por 100 de la cuota del mercado nacional. Le sigue en importancia Valencia, con el 6,6; seguida de Bilbao y Sevilla, con más de 3.500 unidades cada una, representando las tres, en su conjunto, el 14 por 100 , casi la sexta parte de la cuota total de mercado.

Las Zonas de La Coruna, Oviedo, San Sebastián, Zaragoza, Gerona, Alicante, Murcia, Baleares y Málaga, con de dos mil a tres mil unidades cada una, suman el 21,4 por 100 , más de la quinta parte de la cuota nacional de mercado.

Y finalmente, Pontevedra, León, Santander y Pamplona, al Norte; Valladolid y Badajoz, en la Meseta; Lérida, Tarragona y Castellón, en Cataluña y el litoral oriental mediterráneo; Cádiz, Córdoba y Granada, en Andalucía, y Las Palmas y Santa Cruz de Tenerife, en Canarias, con de mil a dos mil unidades cada una, representan el 19,1 por 100 , casi la quinta parte de la cuota de mercado total.

En resumen, 28 Zonas comerciales absorben el 87,1 por 100 de la cuota de mercado nacional, corroborándose el predominio de la distribución periférica y septentrional de la cuota de mercado, en sus principales y máximos valores.

3. La VARIACIÓN DE LA CUOTA DE MERCAdo EN LAS AREAS COMERCIALES EN EL PERIODO 1969-1979

Teniendo en cuenta la cuota de mercado de cada una de las Areas comerciales en los años 1969 y 1979, se ha obtenido la varia- 
ción registrada en cada una de ellas en el periodo comprendido entre ambos años, en tantos por 100.000. Los valores correspondientes son:

\section{Areas}

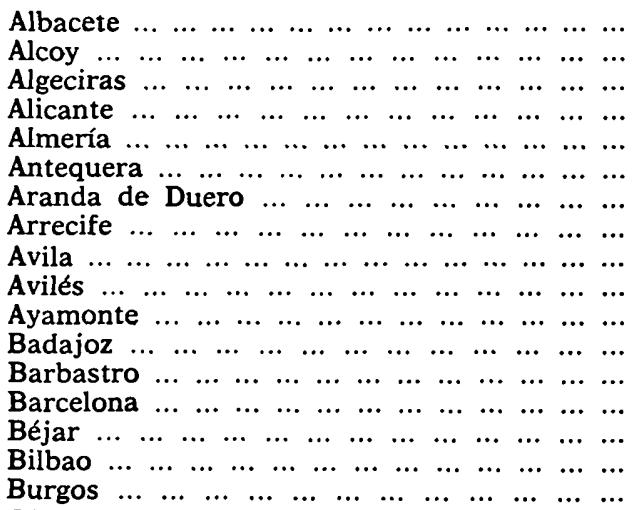

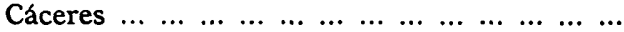

$\begin{array}{llllllllllllll}C a ́ d i z & \ldots & \ldots & \ldots & \ldots & \ldots & \ldots & \ldots & \ldots & \ldots & \ldots & \ldots & \ldots & \ldots\end{array}$

$\begin{array}{llllllllllllll}\text { Calatayud } & \ldots & \ldots & \ldots & \ldots & \ldots & \ldots & \ldots & \ldots & \ldots & \ldots & \ldots & \ldots\end{array}$

$\begin{array}{llllllllllllll}\text { Cartagena } & \ldots & \ldots & \ldots & \ldots & \ldots & \ldots & \ldots & \ldots & \ldots & \ldots & \ldots & \ldots\end{array}$

$\begin{array}{lllllllllllllll}\text { Castellón } & \ldots & \ldots & \ldots & \ldots & \ldots & \ldots & \ldots & \ldots & \ldots & \ldots & \ldots & \ldots\end{array}$

$\begin{array}{lllllllllllllllll}\text { Ciudadela } & \ldots & \ldots & \ldots & \ldots & \ldots & \ldots & \ldots & \ldots & \ldots & \ldots & \ldots & \ldots\end{array}$

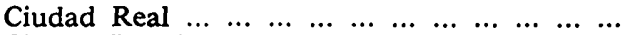

$\begin{array}{lllllllllll}\text { Ciudad Rodrigo } & \ldots & \ldots & \ldots & \ldots & \ldots & \ldots & \ldots & \ldots & \ldots & \ldots\end{array}$

$\begin{array}{llllllllllllllllll}\text { Cordoba } & \ldots & \ldots & \ldots & \ldots & \ldots & \ldots & \ldots & \ldots & \ldots & \ldots & \ldots & \ldots & \ldots\end{array}$

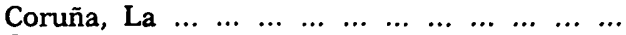

$\begin{array}{lllllllllllllllll}\text { Cuenca } & . . & \ldots & \ldots & \ldots & \ldots & \ldots & \ldots & \ldots & \ldots & \ldots & \ldots & \ldots & \ldots\end{array}$

Don Benito-Villanueva de la Serena ... $\ldots$...

$\begin{array}{lllllllllllllll}\text { Elche } & \ldots & \ldots & \ldots & \ldots & \ldots & \ldots & \ldots & \ldots & \ldots & \ldots & \ldots & \ldots & \ldots\end{array}$

El Ferrol del Caudillo $\ldots \begin{array}{llllllllll} & \ldots & \ldots & \ldots & \ldots & \ldots & \ldots & \ldots & \ldots\end{array}$

$\begin{array}{llllllllllllll}\text { Figueras } & \ldots & \ldots & \ldots & \ldots & \ldots & \ldots & \ldots & \ldots & \ldots & \ldots & \ldots & \ldots\end{array}$

$\begin{array}{lllllllllllllllll}\text { Gandía } & \ldots & \ldots & \ldots & \ldots & \ldots & \ldots & \ldots & \ldots & \ldots & \ldots & \ldots & \ldots & \ldots\end{array}$

$\begin{array}{llllllllllllllll}\text { Gerona } & \ldots & \ldots & \ldots & \ldots & \ldots & \ldots & \ldots & \ldots & \ldots & \ldots & \ldots & \ldots & \ldots\end{array}$

$\begin{array}{lllllllllllllll}\text { Gijón } & \ldots & \ldots & \ldots & \ldots & \ldots & \ldots & \ldots & \ldots & \ldots & \ldots & \ldots & \ldots & \ldots & \ldots\end{array}$

$\begin{array}{llllllllllllll}\text { Granada } & \ldots & \ldots & \ldots & \ldots & \ldots & \ldots & \ldots & \ldots & \ldots & \ldots & \ldots & \ldots\end{array}$

$\begin{array}{llllllllllllll}\text { Guadalajara } & \ldots & \ldots & \ldots & \ldots & \ldots & \ldots & \ldots & \ldots & \ldots & \ldots & \ldots\end{array}$

$\begin{array}{llllllllllllllllll}\text { Huelva } & \ldots & \ldots & \ldots & \ldots & \ldots & \ldots & \ldots & \ldots & \ldots & \ldots & \ldots & \ldots & \ldots\end{array}$

$\begin{array}{llllllllllllllll}\text { Huesca } & \ldots & \ldots & \ldots & \ldots & \ldots & \ldots & \ldots & \ldots & \ldots & \ldots & \ldots & \ldots & \ldots\end{array}$

$\begin{array}{llllllllllllll}\text { Ibiza } & \ldots & \ldots & \ldots & \ldots & \ldots & \ldots & \ldots & \ldots & \ldots & \ldots & \ldots & \ldots & \ldots\end{array}$

$\begin{array}{llllllllllllllllll}\text { Jaca } & \ldots & \ldots & \ldots & \ldots & \ldots & \ldots & \ldots & \ldots & \ldots & \ldots & \ldots & \ldots & \ldots & \ldots\end{array}$

$\begin{array}{lllllllllllllllll} & \text { Jaén } & \ldots & \ldots & \ldots & \ldots & \ldots & \ldots & \ldots & \ldots & \ldots & \ldots & \ldots & \ldots & \ldots & \ldots\end{array}$

$\begin{array}{llllllllllllll}\text { Játiva } & \ldots & \ldots & \ldots & \ldots & \ldots & \ldots & \ldots & \ldots & \ldots & \ldots & \ldots & \ldots & \ldots\end{array}$

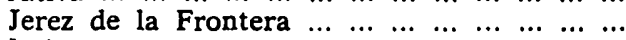

$\begin{array}{llllllllllllll}\text { Leb́n } & \ldots & \ldots & \ldots & \ldots & \ldots & \ldots & \ldots & \ldots & \ldots & \ldots & \ldots & \ldots & \ldots\end{array}$

$\begin{array}{llllllllllllll}\text { Lérida } & \ldots & \ldots & \ldots & \ldots & \ldots & \ldots & \ldots & \ldots & \ldots & \ldots & \ldots & \ldots\end{array}$

$\begin{array}{llllllllllllllll}\text { Linares } & \ldots & \ldots & \ldots & \ldots & \ldots & \ldots & \ldots & \ldots & \ldots & \ldots & \ldots & \ldots & \ldots\end{array}$

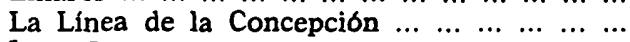

$\begin{array}{lllllllllllllll}\text { Logroño } & \ldots & \ldots & \ldots & \ldots & \ldots & \ldots & \ldots & \ldots & \ldots & \ldots & \ldots & \ldots\end{array}$

$\begin{array}{llllllllllllllll}\operatorname{Lorca} & \ldots & \ldots & \ldots & \ldots & \ldots & \ldots & \ldots & \ldots & \ldots & \ldots & \ldots & \ldots & \ldots\end{array}$

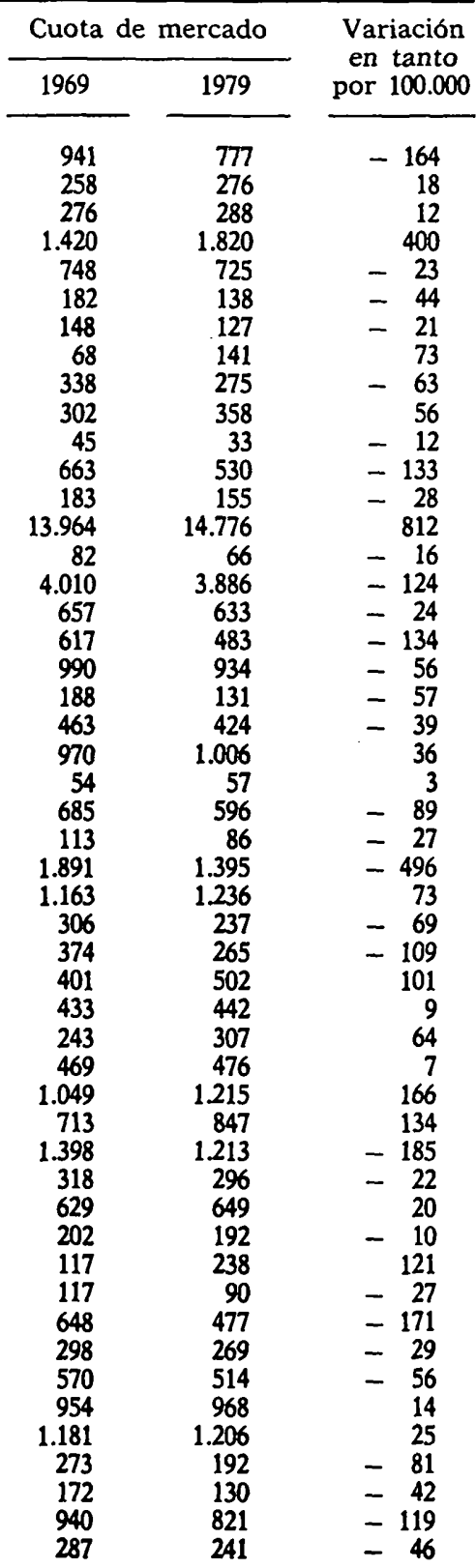




\begin{tabular}{|c|c|c|c|}
\hline \multirow{2}{*}{ Areas } & \multicolumn{2}{|c|}{ Cuota de mercado } & \multirow{2}{*}{$\begin{array}{c}\text { Variación } \\
\text { en tanto } \\
\text { por } 100.000\end{array}$} \\
\hline & 1969 & 1979 & \\
\hline $\begin{array}{llllllllllllll}\text { Lugo } & \ldots & \ldots & \ldots & \ldots & \ldots & \ldots & \ldots & \ldots & \ldots & \ldots & \ldots & \ldots & \ldots\end{array}$ & 848 & 869 & 21 \\
\hline 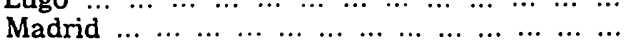 & 15.569 & 16.351 & 782 \\
\hline $\begin{array}{llllllllllllll}\text { Mahón } & \ldots & \ldots & \ldots & \ldots & \ldots & \ldots & \ldots & \ldots & \ldots & \ldots & \ldots & \ldots & \ldots\end{array}$ & 120 & 113 & -7 \\
\hline 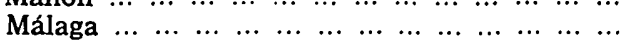 & 1.916 & 2.118 & 202 \\
\hline $\begin{array}{llllllllllllll} & \\
\text { Manresa } & \ldots & \ldots & \ldots & \ldots & \ldots & \ldots & \ldots & \ldots & \ldots & \ldots & \ldots & \ldots & \ldots\end{array}$ & 518 & 562 & 44 \\
\hline $\begin{array}{llllllllllllll}\text { Mérida } & \ldots & \ldots & \ldots & \ldots & \ldots & \ldots & \ldots & \ldots & \ldots & \ldots & \ldots & \ldots & \ldots\end{array}$ & 201 & 172 & 29 \\
\hline 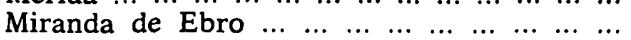 & 144 & 132 & -12 \\
\hline $\begin{array}{lllllllllllllll}\text { Motril } & \ldots & \ldots & \ldots & \ldots & \ldots & \ldots & \ldots & \ldots & \ldots & \ldots & \ldots & \ldots & \ldots & \ldots\end{array}$ & 154 & 146 & -8 \\
\hline $\begin{array}{llllllllllllll}\begin{array}{c}\text { Murcia } \\
\end{array} \ldots & \ldots & \ldots & \ldots & \ldots & \ldots & \ldots & \ldots & \ldots & \ldots & \ldots & \ldots & \ldots & \ldots\end{array}$ & 1.623 & 1.447 & -176 \\
\hline 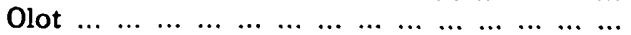 & 130 & 144 & 14 \\
\hline $\begin{array}{lllllllllllll}\text { Onteniente } & \ldots & \ldots & \ldots & \ldots & \ldots & \ldots & \ldots & \ldots & \ldots & \ldots & \ldots\end{array}$ & 114 & 117 & 3 \\
\hline $\begin{array}{lllllllllllllll}\text { Orense } & \ldots & \ldots & \ldots & \ldots & \ldots & \ldots & \ldots & \ldots & \ldots & \ldots & \ldots & \ldots & \ldots\end{array}$ & 836 & 883 & 47 \\
\hline $\begin{array}{lllllllllllllll}\text { Oviedo } & \ldots & \ldots & \ldots & \ldots & \ldots & \ldots & \ldots & \ldots & \ldots & \ldots & \ldots & \ldots & \ldots\end{array}$ & 1.704 & 1.658 & -46 \\
\hline $\begin{array}{lllllllllllll}\text { Palencia } & \ldots & \ldots & \ldots & \ldots & \ldots & \ldots & \ldots & \ldots & \ldots & \ldots & \ldots & \ldots\end{array}$ & 627 & 486 & -141 \\
\hline $\begin{array}{l}\text { Palma } \text { de } \text { Mallorca } \\
\ldots\end{array}$ & 1.744 & 1.889 & 145 \\
\hline $\begin{array}{lllllllllllll}\text { Palmas, } L a s & \ldots & \ldots & \ldots & \ldots & \ldots & \ldots & \ldots & \ldots & \ldots & \ldots & \ldots\end{array}$ & 1.341 & 1.546 & 205 \\
\hline $\begin{array}{llllllllllllll}\text { Pamplona } & \ldots & \ldots & \ldots & \ldots & \ldots & \ldots & \ldots & \ldots & \ldots & \ldots & \ldots & \ldots\end{array}$ & 1.463 & 1.364 & -99 \\
\hline $\begin{array}{lllllllllllll}\text { Plasencia } & \ldots & \ldots & \ldots & \ldots & \ldots & \ldots & \ldots & \ldots & \ldots & \ldots & \ldots & \ldots\end{array}$ & 284 & 229 & -55 \\
\hline $\begin{array}{llllllllllll}\text { Ponferrada } & \ldots & \ldots & \ldots & \ldots & \ldots & \ldots & \ldots & \ldots & \ldots & \ldots & \ldots\end{array}$ & 387 & 371 & \\
\hline $\begin{array}{lllllllllllll}\text { Pontevedra } & \ldots & \ldots & \ldots & \ldots & \ldots & \ldots & \ldots & \ldots & \ldots & \ldots & \ldots\end{array}$ & 547 & 601 & 54 \\
\hline $\begin{array}{llllllllllllll}\text { Puertollano } & \ldots & \ldots & \ldots & \ldots & \ldots & \ldots & \ldots & \ldots & \ldots & \ldots & \ldots & \ldots & \ldots\end{array}$ & 172 & 143 & \\
\hline $\begin{array}{lllllllllllllll}\text { Puigcerdá } & \ldots & \ldots & \ldots & \ldots & \ldots & \ldots & \ldots & \ldots & \ldots & \ldots & \ldots & \ldots\end{array}$ & 48 & 50 & 2 \\
\hline $\begin{array}{llllllllllllllll}\operatorname{Reus} & \ldots & \ldots & \ldots & \ldots & \ldots & \ldots & \ldots & \ldots & \ldots & \ldots & \ldots & \ldots & \ldots & \ldots\end{array}$ & 481 & 516 & 35 \\
\hline $\begin{array}{llllllllllllll}\boldsymbol{R} \text { Ronda } & \ldots & \ldots & \ldots & \ldots & \ldots & \ldots & \ldots & \ldots & \ldots & \ldots & \ldots & \ldots & \ldots\end{array}$ & 212 & 169 & 43 \\
\hline $\begin{array}{llllllllllll}\text { Salamanca } & \ldots & \ldots & \ldots & \ldots & \ldots & \ldots & \ldots & \ldots & \ldots & \ldots & \ldots\end{array}$ & 849 & 759 & \\
\hline 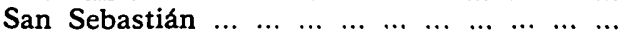 & 2.298 & 2.028 & -270 \\
\hline 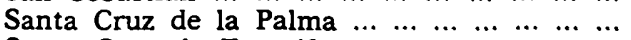 & 132 & 122 & \\
\hline $\begin{array}{llllllll}\text { Santa Cruz de } \text { Tenerife } & \ldots & \ldots & \ldots & \ldots & \ldots & \ldots & \ldots\end{array}$ & 1.217 & 1.362 & 145 \\
\hline $\begin{array}{llllllllllll}\text { Santander } & \ldots & \ldots & \ldots & \ldots & \ldots & \ldots & \ldots & \ldots & \ldots & \ldots & \ldots\end{array}$ & 1.251 & 1.277 & 26 \\
\hline 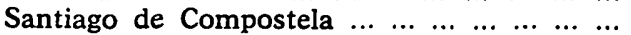 & 682 & 731 & 49 \\
\hline $\begin{array}{lllllllllllll}\text { Segovia } & \ldots & \ldots & \ldots & \ldots & \ldots & \ldots & \ldots & \ldots & \ldots & \ldots & \ldots & \ldots\end{array}$ & 405 & 317 & -88 \\
\hline $\begin{array}{llllllllllllll}\text { Sevilla } & \ldots & \ldots & \ldots & \ldots & \ldots & \ldots & \ldots & \ldots & \ldots & \ldots & \ldots & \ldots & \ldots\end{array}$ & 3.818 & 3.560 & -258 \\
\hline $\begin{array}{lllllllllllllll}\text { Soria } & \ldots & \ldots & \ldots & \ldots & \ldots & \ldots & \ldots & \ldots & \ldots & \ldots & \ldots & \ldots & \ldots\end{array}$ & 280 & 199 & -81 \\
\hline Talavera de la $\operatorname{Reina}\left(\begin{array}{lllllllll} & \ldots & \ldots & \ldots & \ldots & \ldots & \ldots & \ldots & \ldots\end{array}\right.$ & 520 & 484 & -36 \\
\hline $\begin{array}{lllllllllllll}\operatorname{Tarragona} & \ldots & \ldots & \ldots & \ldots & \ldots & \ldots & \ldots & \ldots & \ldots & \ldots & \ldots & \ldots\end{array}$ & 340 & 474 & 134 \\
\hline $\begin{array}{lllllllllllll}\text { Teruel } & \ldots & \ldots & \ldots & \ldots & \ldots & \ldots & \ldots & \ldots & \ldots & \ldots & \ldots & \ldots\end{array}$ & 264 & 197 & -67 \\
\hline $\begin{array}{lllllllllllll}\text { Toledo } & \ldots & \ldots & \ldots & \ldots & \ldots & \ldots & \ldots & \ldots & \ldots & \ldots & \ldots & \ldots\end{array}$ & 391 & 335 & -56 \\
\hline $\begin{array}{lllllllllllll}\text { Tortosa } & \ldots & \ldots & \ldots & \ldots & \ldots & \ldots & \ldots & \ldots & \ldots & \ldots & \ldots & \ldots\end{array}$ & 450 & 393 & -57 \\
\hline $\begin{array}{ccccccccccccc}\text { Ubeda } & \ldots & \ldots & \ldots & \ldots & \ldots & \ldots & \ldots & \ldots & \ldots & \ldots & \ldots & \ldots\end{array}$ & 377 & 249 & -128 \\
\hline $\begin{array}{cccccccccccccc}\text { Utiel } & \ldots & \ldots & \ldots & \ldots & \ldots & \ldots & \ldots & \ldots & \ldots & \ldots & \ldots & \ldots & \ldots\end{array}$ & 87 & 64 & -23 \\
\hline $\begin{array}{lllllllllllll}\text { Valdepeñas } & \ldots & \ldots & \ldots & \ldots & \ldots & \ldots & \ldots & \ldots & \ldots & \ldots & \ldots & \ldots\end{array}$ & 167 & 130 & -37 \\
\hline $\begin{array}{lllllllllllll}\text { Valencia } & \ldots & \ldots & \ldots & \ldots & \ldots & \ldots & \ldots & \ldots & \ldots & \ldots & \ldots & \ldots\end{array}$ & 4.858 & 5.132 & 274 \\
\hline 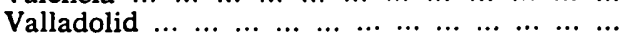 & 1.178 & 1.200 & 22 \\
\hline $\begin{array}{lllllllllllllll}V \text { Valls } & \ldots & \ldots & \ldots & \ldots & \ldots & \ldots & \ldots & \ldots & \ldots & \ldots & \ldots & \ldots & \ldots & \ldots\end{array}$ & 110 & 106. & \\
\hline 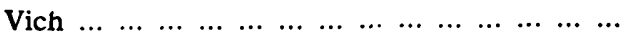 & 365 & 369 & 4 \\
\hline $\begin{array}{llllllllllllll}\text { Vigo } & \ldots & \ldots & \ldots & \ldots & \ldots & \ldots & \ldots & \ldots & \ldots & \ldots & \ldots & \ldots & \ldots\end{array}$ & 946 & 1.025 & 79 \\
\hline $\begin{array}{lllllllll}\text { Villafranca del Penedés } & \ldots & \ldots & \ldots & \ldots & \ldots & \ldots & \ldots\end{array}$ & 226 & 290 & 64 \\
\hline $\begin{array}{llllllllllllll}\text { Vitoria } & \ldots & \ldots & \ldots & \ldots & \ldots & \ldots & \ldots & \ldots & \ldots & \ldots & \ldots & \ldots & \ldots\end{array}$ & 623 & 820 & 197 \\
\hline $\begin{array}{lllllllllllllll}\operatorname{Zafra} & \ldots & \ldots & \ldots & \ldots & \ldots & \ldots & \ldots & \ldots & \ldots & \ldots & \ldots & \ldots & \ldots\end{array}$ & 283 & 182 & -101 \\
\hline $\begin{array}{lllllllllllll}\text { Zamora } & \ldots & \ldots & \ldots & \ldots & \ldots & \ldots & \ldots & \ldots & \ldots & \ldots & \ldots & \ldots\end{array}$ & 557 & 462 & -95 \\
\hline $\begin{array}{lllllllllllll}Z \text {Zaragoza} & \ldots & \ldots & \ldots & \ldots & \ldots & \ldots & \ldots & \ldots & \ldots & \ldots & \ldots & \ldots\end{array}$ & 2.632 & 2.518 & -114 \\
\hline
\end{tabular}


En líneas generales, se registra un incremento de la cuota de mercado, en el período considerado, en 41 Areas comerciales, y, consiguientemente, una disminución en 60 de ellas.

La distribución geográfica de las Areas comerciales, cuya cuota de mercado se ha incrementado, configura las siguientes Regiones:

Primera: Noroeste. Comprende las Areas comerciales de La Coruña, Santiago de Compostela, El Ferrol, Lugo, Pontevedra, Vigo, Orense, Avilés, Gijón y León, y se extiende a Santander y Vitoria, abarcando toda Galicia, Asturias, la Provincia de León, Cantabria y Alava, a excepción de las Areas de Oviedo, Ponferrada y Miranda de Ebro, en las que la cuota de mercado decreció en dicho período. Los incrementos máximos corresponden a las Areas de Vitoria y Gijón, y en todas las demás el incremento es moderado, salvo en la de El Ferrol, cuyo aumento es tan reducido que más bien refleja una situación estacionaria.

Segunda: Cataluña. Abarca las Areas de Lérida, Puigcerdá, Gerona, Figueras, Olot, Vich, Barcelona, Manresa, Villafranca del Penedés, Tarragona y Reus, es decir, toda Cataluña, con excepción de las Areas de Valls y Tortosa, cuya cuota de mercado ha disminuido. El incremento máximo corresponde al Area de Barcelona, la primera de España, y son de destacar también los incrementos registrados en las Areas de Gerona y Tarragona; el incremento es moderado en las demás Areas, muy reducido en la de Olot y prácticamente inexistente en las de Puigcerdá y Vich.

Tercera: Central. En esta Región sólo se registra el incremento extraordinario del Area de Madrid, la segunda de España, a continuación de la de Barcelona, y el realmente reducido de la de Valladolid.

Cuarta: Región Valenciana. Comprende las Areas de Castellón, Valencia, Gandia, Onteniente, Alicante, Alcoy y Elche, es decir, toda la Región, con excepción de las Areas de Játiva y Utiel, cuya cuota de mercado decrece. Los incrementos registrados por las Areas de Alicante y Valencia, muy importantes, las sitúan en tercero y cuarto lugares, respectivamente, después de las de Barcelona y Madrid, e igualmente destaca el incremento de Elche; es muy reducido el de Alcoy, prácticamente inexistente en las de Gandía y Onteniente y moderado en las restantes.

Quinta: Andalucía. Comprende tan sólo las Areas de Málaga, Huelva y Algeciras, únicas que registran incrementos en sus cuotas 
de mercado. Muy importante es el incremento de la primera, que ocupa el sexto lugar entre las Areas comerciales españolas, mientras que el de las otras dos es muy reducido.

Sexta: Baleares. Abarca las Areas de Palma de Mallorca, Ibiza y Ciudadela, con incrementos importantes en las dos primeras y prácticamente inexistente en la última. Tan sólo disminuye la cuota de mercado del Area de Mahón.

Séptima: Canarias. Comprende las Areas de Santa Cruz de Tenerife, Arrecife y Las Palmas, con excepción de la de Santa Cruz de la Palma, cuya cuota de mercado decrece. Importante es el incremento registrado en las de las dos capitales canarias, en especial el de Las Palmas, que ocupa el quinto lugar entre las Areas comerciales españolas, y es de destacar también el incremento de la cuota de mercado en el Area de Arrecife.

En resumen de lo expuesto puede afirmarse que, con un incremento superior, a lo menos, a 100 unidades, superando en un caso las 800 , lo que equivale del 1 al 8 por 1.000 , figuran, por orden de importancia, las Areas de Barcelona, Madrid, Alicante, Valencia, Las Palmas, Málaga, Vitoria, Gerona, Palma de Mallorca, Santa Cruz de Tenerife, Gijón, Tarragona, Ibiza y Elche, en total 14, situadas geográficamente: dos al Norte, una en el Centro de España, ocho en el litoral oriental mediterráneo y balear, una en Andalucía y dos en Canarias, corroborando, una vez más, el carácter predominantemente periférico del desarrollo de la cuota de mercado de las principales Areas comerciales españolas.

Por el contrario, la disminución de la cuota de mercado, que, como hemos dicho, afecta a 60 Areas, resulta más acusada, superior al 1 por 1.000, en las 16 siguientes: Bilbao y San Sebastián, al Norte; Logroño y Zaragoza, en el Valle del Ebro; Palencia, Cáceres y Badajoz, Don Benito-Villanueva de la Serena y Zafra, así como Albacete, en la Meseta; Murcia, Jaén, Granada, Ubeda, Sevilla y Córdoba, al Sur. Es de señalar el hecho de que la tendencia decreciente de la cuota de mercado se manifiesta en Areas cuyas capitales son algunas de las principales ciudades españolas, de manera singular las dos primeramente reseñadas. 


\section{LA CUOTA DE MERCADO PER CÁPITA DE LAS AREAS COMERCIALES EN 1979}

Como complemento de lo expuesto en los apartados anteriores, parece interesante contemplar y analizar la cuota de mercado per cápita de las Areas comerciales en dicho año.

Con un valor positivo, superior a la unidad, figuran las siguientes Areas:

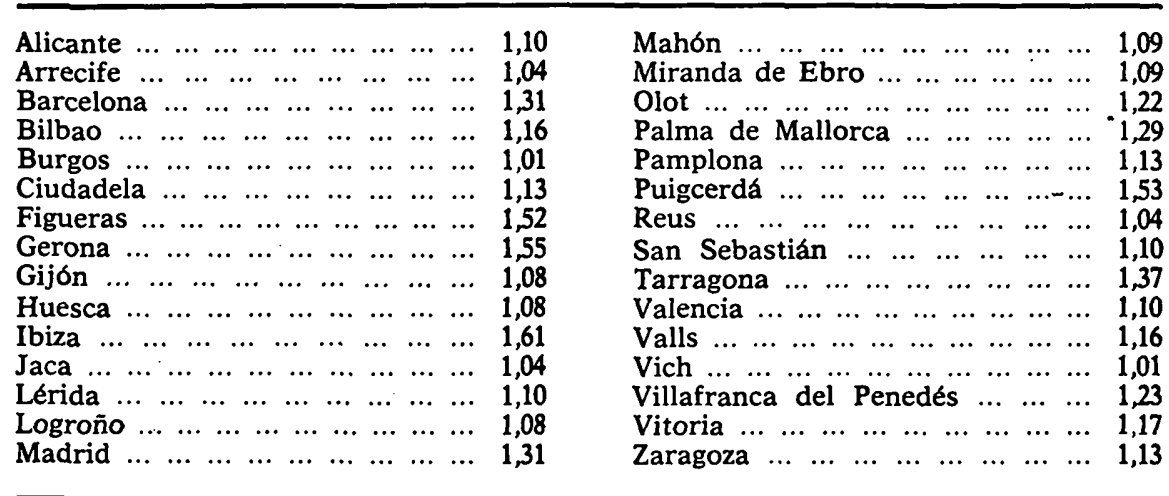

Con valores inferiores a la unidad figuran, a su vez, estas Areas:

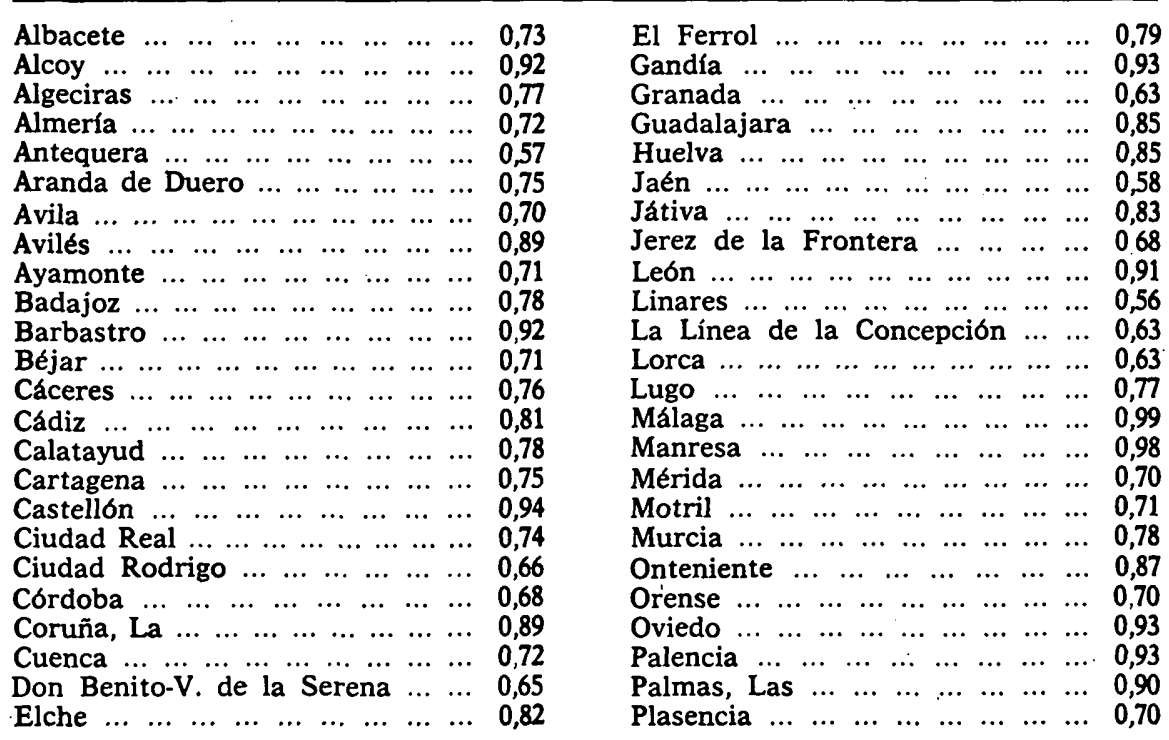




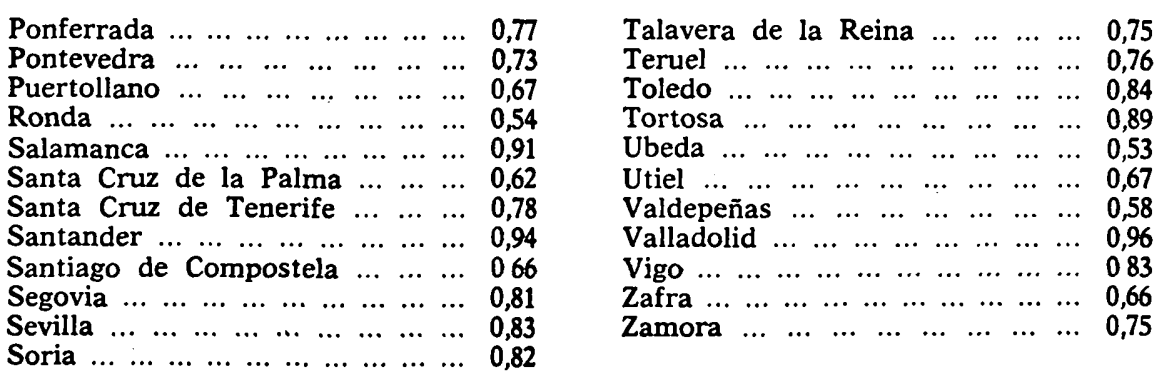

La distribución geográfica de las 30 Areas comerciales con una cuota de mercado per cápita superior a la unidad presenta ciertas analogías, a la par que marcadas diferencias, con las Regiones consideradas en la distribución de las Areas cuya cuota de mercado se incrementó en el período 1969-1979.

De la Región Noroeste, tan sólo destacan Gijón y Vitoria, y a ellas se suman, al Norte, Bilbao y San Sebastián, y varias Areas del Valle del Ebro: Miranda, Pamplona, Logroño, Huesca, Jaca y Zaragoza, abarcando estas cuotas de mercado per cápita positivas las Vascongadas y Navarra, así como Aragón, con la excepción de las Areas de Barbastro, Calatayud y Teruel.

Hay una fuerte coincidencia en Cataluña y Baleares. Todas las Areas comerciales catalanas -salvo Manresa y Tortosa- y las de las Baleares destacan por sus altas cuotas de mercado per cápita y entre ellas se hallan los valores máximos.

En la Región Central destacan tan sólo Madrid y Burgos, y en la Región Valenciana, Alicante y Valencia solamente. No figura en este grupo ninguna Area andaluza, y entre las canarias, solamente Arrecife.

Las cuotas de mercado per cápita de las 71 Areas con valores inferiores a la unidad, oscilan entre 0,53 y 0,99 . Las 13 Areas con valores cercanos a la unidad y superiores a 0,91 se sitúan preferentemente al Norte, en el Valle del Duero y en la Región Valenciana, sin perjuicio de que cuatro de ellas se hallen en el Valle del Ebro, Cataluña, Andalucía y Canarias.

Las 14 Areas con valores inmediatamente inferiores, por encima de 0,81 , se distribuyen, en forma proporcional, en el Noroeste, en ambas vertientes del Sistema Central, en la Región Valenciana y en Andalucía, salvo una de ellas catalana. 
A 26 asciende el número de Areas del grupo siguiente, con valores superiores a 0,71 , que se sitúan primordialmente en Galicia, ambas Submesetas y Andalucía, salvo cinco de ellas situadas en el Valle del Ebro, Murcia y Canarias.

Otras 12 Areas, con valores entre 0,61 y 070 , se hallan, sobre todo, en ambas Submesetas y Andalucía, a excepción de cuatro sitas en Galicia, la Región Valenciana, Murcia y Canarias.

Finalmente, los valores mínimos corresponden a seis Areas sitas en Andalucía, salvo una en la Submeseta Sur.

De lo expuesto en los párrafos anteriores se deduce una tendencia hacia el desplazamiento de los valores de la cuota de mercado per cápita de Norte a Sur, en forma progresiva, correspondiendo, en general, los valores máximos al Norte y los mínimos al Sur.

\section{LA CUOTA DE RIQUEZA ACTIVA Y EL NIVEL DE DESARROLLO DE LAS AREAS COMERCIALES ESPAÑOLAS}

Como complemento de lo expuesto respecto a la cuota de mercado, parece oportuno abordar un ligero análisis de la cuota de riqueza activa y del nivel de desarrollo de las Areas comerciales españolas, con referencia al año 1977, último del que se dispone de datos elaborados.

Por riqueza activa se entiende el conjunto de elementos que, inmersos en la actividad económica del país, contribuyen al proceso de desarrollo y a la formación de la renta nacional.

La cuota de riqueza activa se deduce de tres índices: demográfico, cultural y económico.

El índice demográfico se basa en los datos municipales de población según el Padrón renovado de 1975; de población urbana estimada aplicando las proporciones del Censo de 1970 a la población de cada Municipio en 1975, y en la cifra de inmigrantes recibidos en un año, según estimaciones realizadas a nivel de Area. Estos tres componentes del índice vienen a expresar el volumen de la población, la forma de su distribución sobre el territorio y la fuerza atractiva de sus núcleos.

El índice cultural se apoya en dos datos: el número de alumnos de Bachillerato y los cursos quinto a octavo, ambos inclusive, de 
E. G. B., y el número de editoriales, librerías de nuevo o usado y puestos fijos o quioscos que, tributando como librerías, se dedican a la venta de libros.

El índice económico es el resultado de un índice general de riqueza, deducido de los indicadores basados en las estimaciones valorativas de la riqueza agrícola, de la riqueza urbana y de la riqueza industrial y comercial, y de la cifra de camiones y teléfonos existentes en cada Area.

A cada una de las series, enumeradas en los párrafos anteriores, como componentes de los índices demográfico, cultural y económico, se le asigna un valor con referencia a 100.000 unidades como total nacional de cada serie. Cada índice se obtiene como media aritmética ponderada de las series de datos en que se basan, y de igual forma se obtiene la cuota de riqueza activa sobre los índices demográfico, cultural y económico.

El nivel de desarrollo de cada Area es la relación existente entre la cuota de riqueza activa y el índice de su población total, considerando 100 el nivel medio nacional.

La cuota de riqueza activa y el nivel de desarrollo correspondientes a cada Area son los siguientes:

\begin{tabular}{|c|c|c|}
\hline Areas & $\begin{array}{c}\text { Cuota } \\
\text { de riqueza } \\
\text { activa }\end{array}$ & $\begin{array}{l}\text { Nivel de } \\
\text { desarrollo }\end{array}$ \\
\hline 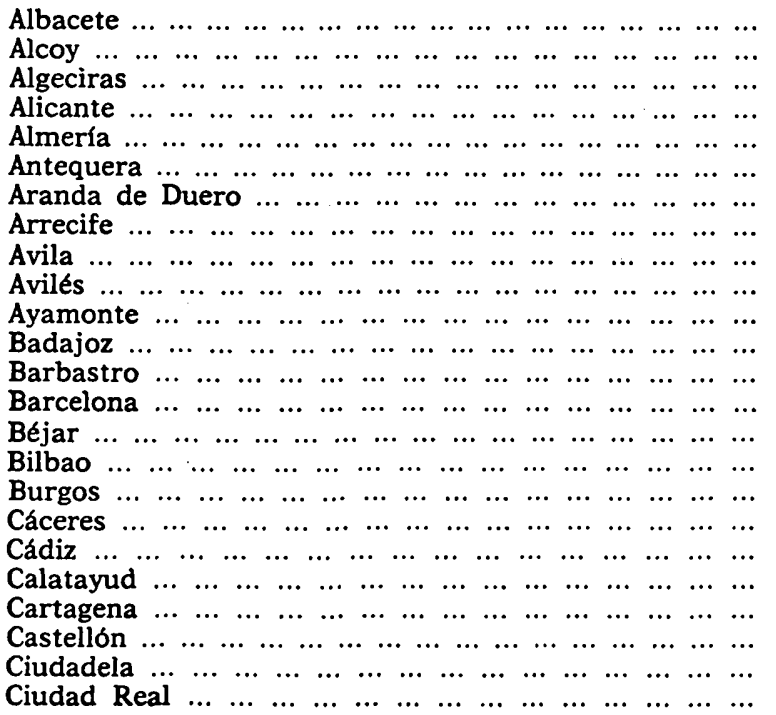 & $\begin{array}{r}829,7 \\
382,2 \\
370,4 \\
1.793,7 \\
884,1 \\
168,6 \\
141,1 \\
155,0 \\
357,2 \\
444,2 \\
32,2 \\
598,8 \\
180,9 \\
13.788,6 \\
80,5 \\
3.830,8 \\
643,5 \\
505,5 \\
1.031,1 \\
128,9 \\
626,9 \\
1.081,9 \\
732 \\
658,7\end{array}$ & $\begin{array}{r}78,0 \\
127,8 \\
99,3 \\
108,7 \\
87,4 \\
69,1 \\
83,7 \\
114,2 \\
90,4 \\
110,6 \\
68,8 \\
88,0 \\
107,9 \\
122,7 \\
86,6 \\
114,1 \\
1025 \\
79,5 \\
89,9 \\
76,3 \\
110,9 \\
100,8 \\
145,5 \\
81,5\end{array}$ \\
\hline
\end{tabular}




\begin{tabular}{|c|c|c|}
\hline Areas & $\begin{array}{l}\text { Cuota } \\
\text { de riqueza } \\
\text { activa }\end{array}$ & $\begin{array}{l}\text { Nivel de } \\
\text { desarrollo }\end{array}$ \\
\hline 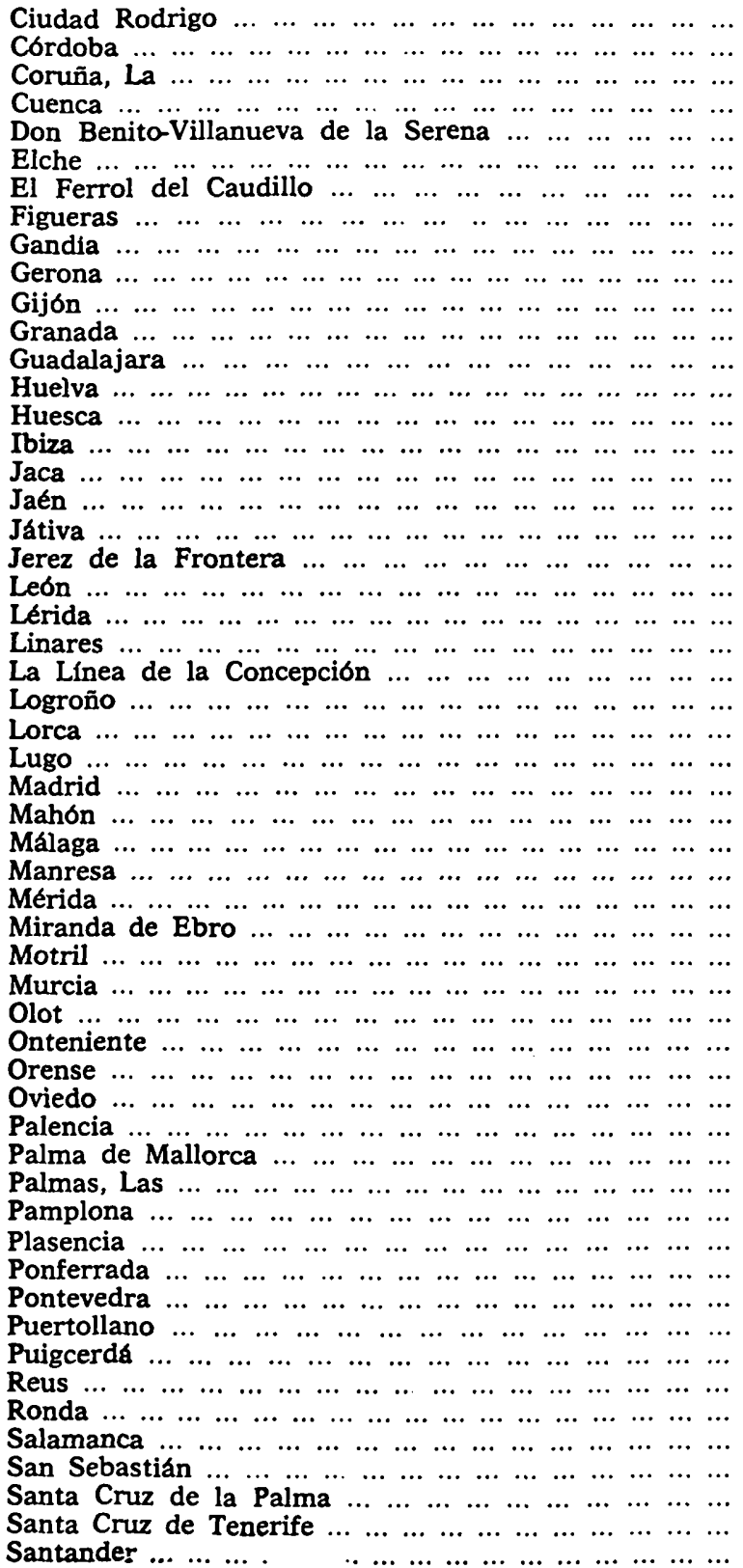 & $\begin{array}{r}80,9 \\
1.615,6 \\
1.172,1 \\
280,3 \\
275,4 \\
587,3 \\
438,4 \\
243,0 \\
463,6 \\
951,5 \\
820,6 \\
1.632,0 \\
438,8 \\
647,8 \\
179,6 \\
164,3 \\
87,7 \\
652,0 \\
267,9 \\
600,4 \\
914,6 \\
970,9 \\
249,9 \\
211,3 \\
703,8 \\
269,5 \\
747,3 \\
16.055,2 \\
121,3 \\
2.144,0 \\
529,5 \\
184,1 \\
126,8 \\
159,1 \\
1.560,3 \\
117,5 \\
131,2 \\
735,1 \\
1.442,2 \\
527,7 \\
1.624,0 \\
1.800,7 \\
1.316,7 \\
225,9 \\
333,5 \\
540,5 \\
238,4 \\
41,2 \\
600,5 \\
17,5 \\
75,8 \\
2.008,7 \\
164,6 \\
1.418,1 \\
1253,9\end{array}$ & $\begin{array}{r}62,2 \\
78,2 \\
84,0 \\
84,7 \\
67,1 \\
96,4 \\
78,7 \\
120,2 \\
90,7 \\
121,5 \\
104,5 \\
85,3 \\
116,5 \\
85,2 \\
101,2 \\
111,2 \\
101,6 \\
79,1 \\
83,1 \\
79,7 \\
86,3 \\
88,4 \\
73,4 \\
102,6 \\
92,5 \\
70,2 \\
65,9 \\
128,6 \\
117,3 \\
100,0 \\
92,6 \\
74,9 \\
104,7 \\
77,0 \\
84,2 \\
99,2 \\
97,5 \\
58,6 \\
81,1 \\
101,0 \\
110,8 \\
105,4 \\
108,7 \\
69,0 \\
69,5 \\
65,9 \\
112,4 \\
126,4 \\
120,9 \\
56,8 \\
89,6 \\
108,7 \\
84,3 \\
81,5 \\
92,3\end{array}$ \\
\hline
\end{tabular}




\begin{tabular}{|c|c|c|}
\hline Areas & $\begin{array}{c}\text { Cuota } \\
\text { de riqueza } \\
\text { activa }\end{array}$ & $\begin{array}{l}\text { Nivel de } \\
\text { desarrollo }\end{array}$ \\
\hline 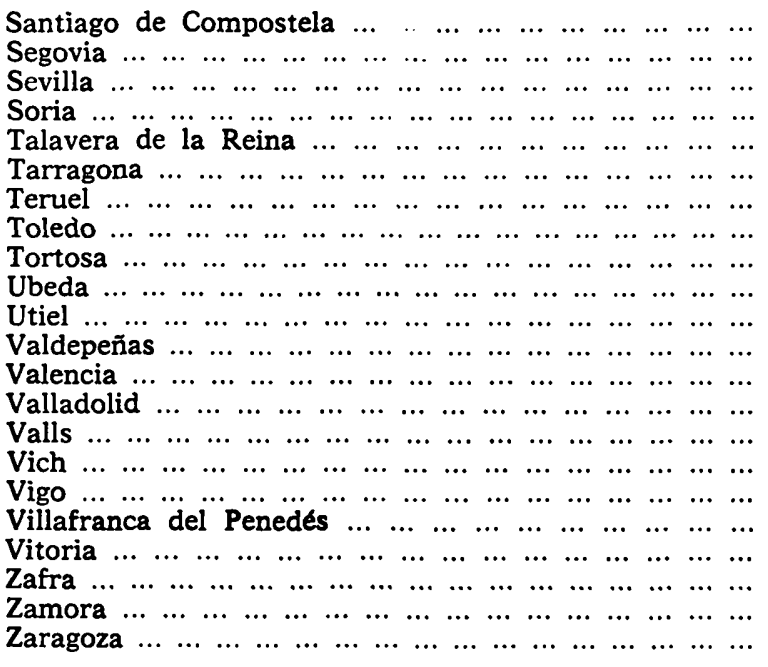 & $\begin{array}{r}562,1 \\
409,9 \\
3.5633 \\
210,4 \\
524,2 \\
415,2 \\
208,1 \\
392,8 \\
388,0 \\
266,3 \\
63,9 \\
168,1 \\
5.159,3 \\
1.259,8 \\
1061 \\
368,3 \\
1.010,5 \\
234,5 \\
927,3 \\
165,9 \\
465,8 \\
2.347,7\end{array}$ & $\begin{array}{r}50,8 \\
104,3 \\
82,8 \\
86,8 \\
81,1 \\
119,8 \\
80,4 \\
98,5 \\
883 \\
56,7 \\
67,1 \\
74,5 \\
110,9 \\
100,4 \\
115,8 \\
100,7 \\
81,4 \\
99,7 \\
132,4 \\
59,7 \\
754 \\
105,4\end{array}$ \\
\hline
\end{tabular}

Del examen de las cifras anteriores se deduce, en primer término, la existencia de un marcado paralelismo entre la cuota de riqueza activa en 1977 y la cuota de mercado en 1979.

En ambas figuran a la cabeza las Areas de Madrid y Barcelona, si bien entre ambas cubren el 29,8 por 100 de la cuota de riqueza activa, mientras alcanzaban el 31,1 por 100 de la cuota de mercado.

Valencia, con valores similares, y juntamente con Bilbao y San Sebastián, Zaragoza, Sevilla y Málaga, suman valores idénticos, representando la quinta parte de ambas cuotas.

$\mathrm{Y}$ finalmente, con cuotas de riqueza activa superior a mil unidades figuran 15 Areas, en gran parte coincidentes con las de análogo nivel en cuanto a la cuota de mercado. La Coruña, Vigo, Oviedo, Santander y Pamplona, al Norte; Valladolid, en la Submeseta Norte; Castellón, Alicante, Murcia y Palma de Mallorca - no así Lérida y Gerona, con cuota de riqueza activa inferior a la de mercadoen el litoral mediterráneo; Córdoba y Granada, más Cádiz, en Andalucía, y las de las dos capitales canarias, absorbiendo, en conjunto, más de la quinta parte de la cuota de riqueza activa. En resumen, las 23 Areas reseñadas retienen, en conjunto, el 74,9 por 100, o sea, las tres cuartas partes de la cuota de riqueza activa. 
Las Areas con cuotas mínimas de mercado y de riqueza activa coinciden plenamente, y son Ayamonte, Béjar, Ciudadela, Ciudad Rodrigo, Jaca, Puigcerdá y Utiel, con valores inferiores a 100 unidades.

Las Areas comerciales cuyo nivel de desarrollo es superior al nacional, cifrado en 100, son 39, y entre ellas destaca, en primer lugar, Ciudadela, figurando a continuación Vitoria, Alcoy, Barcelona, Figueras, Gerona, Madrid, Puigcerdá, Mahón y Tarragona, siendo de resaltar el predominio de las catalanas y baleáricas, con la excepción de Vitoria y de Madrid, y el nivel ligeramente superior de Madrid sobre Barcelona.

La distribución geográfica de las Areas según su nivel de desarrollo se asemeja bastante a la correspondiente a las Areas cuya cuota de mercado se ha incrementado en el período 1969-1979, con algunos matices diferenciales.

En la Región Norte sobresalen: Avilés y Gijón, Bilbao y San Sebastián, Vitoria y Miranda de Ebro, Pamplona, Jaca, Huesca y Barbastro, y Zaragoza, abarcando la orla cantábrica, salvo Santander y gran parte del Valle del Ebro aragonés.

En la Meseta destacan Palencia, Burgos, Valladolid y Segovia en el Valle del Duero, y Madrid, Guadalajara y Puertollano en la Submeseta Sur.

Se da nuevamente una fuerte coincidencia en Cataluña y $\mathrm{Ba}$ leares, pues resaltan por su nivel de desarrollo todas las Areas baleáricas y Gerona, Figueras, Puigcerdá, Vich, Barcelona, Tarragona, Reus y Valls.

En la Región Valenciana destacan Castellón, Valencia, Alicante, Alcoy y Cartagena. En Andalucía solamente Málaga y La Línea de la Concepción. Y las de Las Palmas y Arrecife.

Esquemáticamente, el Norte, con el Valle del Duero y el litoral oriental mediterráneo, predominan abiertamente por su mayor nivel de desarrollo; quedan manchas en ambas Submesetas, destacando Madrid, por su singularidad, y se presentan en muy pocos casos en las Regiones del Sur.

Las Areas con valores inferiores pero cercanos al promedio nacional amplían preferentemente y completan las Regiones catalana y valenciana, a excepción de Santander, Avila, Toledo y Algeciras, que se suman a sus respectivas Regiones.

Las Areas del grupo inmediato inferior al anterior, las más numerosas, se distribuyen, en forma casi similar, entre el Norte- 
Noroeste, ambas Submesetas, Cataluña-Valencia y Andalucía, a más de dos canarias, y lo propio ocurre con las Areas con nivel de desarrollo entre 71 y 80 . Los valores mínimos corresponden predominantemente a Areas gallegas, de la Submeseta Sur y andaluzas.

En resumen, se advierte un predominio de los altos niveles de desarrollo al Norte y Este, con progresión de Norte a Sur y hacia el Noroeste de los valores inferiores y mínimos.

Una vez más se aprecia la falta de homogeneidad con que se estructura, distribuye y evoluciona en nuestro país la población y la riqueza, así como los niveles del futuro desarrollo. 


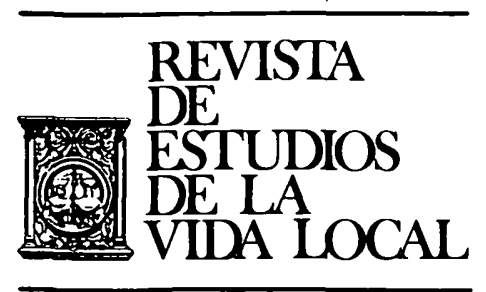

\section{IURISPRUDENCIA}


REVL-1979, núm. 203. BALLESTER ROS, IGNACIO. ALGUNAS CARACTERISTICAS DE LAS AREA... 